\title{
Fasudil inhibits lung carcinoma-conditioned endothelial cell viability and migration
}

\author{
ZHE ZHANG ${ }^{1,2}$, JING-HUA REN ${ }^{2}$, ZHEN-YU LI $^{2}$, LI NONG $^{2}$ and GANG WU ${ }^{2}$ \\ ${ }^{1}$ Department of Integrated Therapy, Fudan University Shanghai Cancer Center; Department of Oncology, \\ Shanghai Medical College, Fudan University, Shanghai 200032; ${ }^{2}$ Department of Oncology, \\ The Union Hospital, Tongji Medical College, Huazhong University of Science and Technology, Wuhan 430022, P.R. China
}

Received November 11, 2011; Accepted January 17, 2012

DOI: $10.3892 / o r .2012 .1686$

\begin{abstract}
The aim of this study was to investigate the effect of fasudil on lung carcinoma-conditioned endothelial cells (LCc-ECs). To obtain LCc-ECs, human umbilical vein endothelial cells (HUVECs) were treated with conditioned cell culture media from human A549 lung adenocarcinoma cells. The effect of fasudil on the viability of LCc-ECs was assessed using the MTT assay, in vitro invasive ability was evaluated using the transwell chamber assay and cytoskeletal changes were detected using fluorescein-labelled phalloidin immunocytochemistry. RhoA mRNA and p-MLC protein expression were measured using RT-PCR and western blotting. Fasudil significantly and dose-dependently inhibited LCc-EC proliferation and in vitro invasive ability. Fasudil also led to stress fibre breakage and fracture in LCc-ECs, indicating that fasudil impacts polymerisation of the cytoskeletal actin filament network. Expression of RhoA mRNA and protein expression of the ROCK substrate p-MLC were reduced by fasudil, suggesting that fasudil can inhibit RhoA/ROCK signalling and attenuate angiogenesis in LCc-ECs. This study indicates that fasudil is an anti-angiogenic agent with potential application for the treatment of cancer, especially lung adenocarcinoma.
\end{abstract}

\section{Introduction}

Angiogenesis is the formation of new capillary blood vessels from endothelial or stem cells, and is essential during wound healing, embryonic development and tumorigenesis (1). Angiogenesis is intricately regulated by a number of factors, including the extracellular matrix, growth factors, membranebound proteinases and integrins which induce cytoskeleton rearrangement and delicately orchestrate the various steps

Correspondence to: Professor Gang Wu, Department of Oncology, The Union Hospital, Tongji Medical College, Huazhong University of Science and Technology, Wuhan 430022, P.R. China

E-mail: try_again_it@yahoo.cn

Key words: fasudil, lung carcinoma-conditioned endothelial cells, stress fibre, human umbilical vein endothelial cells, migration of angiogenesis, including endothelial cell (EC) proliferation, branching and sprouting and lumen formation (2). Cells migrate by rearranging the actin cytoskeleton (3), and formation of adhesive structures and cellular contraction are essential steps during cell migration. The actin cytoskeleton plays a critical role in the regulation of the complex series of signalling events which lead to EC shape changes during migration and angiogenesis.

Tumour and normal endothelium are distinct at the molecular level. St. Croix et al (4) compared the gene expression patterns of endothelial cells derived from the blood vessels of normal and malignant colorectal tissues. Of over 170 transcripts which are predominantly expressed in the endothelium, 79 were differentially expressed, 46 of which, including TEMI and TEM 8 were specifically elevated in the tumour-associated endothelium.

The small GTPase RhoA and the downstream effector, Rho-kinase (ROCK) play a central role in diverse cellular functions, including smooth muscle contraction, cytoskeleton rearrangement, cell migration, cell proliferation and gene expression (5). Rho-kinase regulates stress fibre formation and cell-cell junctions in ECs in response to vascular endothelial derived growth factor (VEGF) (6). Members of the Rho family of GTPases have also emerged as key players in the regulation of a variety of biological activities involving cell motility and actin cytoskeleton reorganization (7). Several members of this family, including RhoA, Cdc42 and Racl are involved in focal adhesion complex assembly, cell polarity, gene transcription and cell-cycle progression (8).

Fasudil (HA-1077), a ROCK inhibitor, has been approved in Japan since 1995 for the clinical treatment of vascular spasms in the brain $(9,10)$. Yin et al (11) showed that fasudil inhibits VEGF-induced angiogenesis in vitro and in vivo. However, the effect of fasudil on lung carcinoma-conditioned endothelial cells (LCc-EC) has not been reported. In this study, we investigated the effect and mechanism of action of fasudil on LCc-EC to explore the potential for the application of fasudil in lung cancer treatment.

\section{Materials and methods}

Lung carcinoma-conditioned endothelial cell culture. The A549 lung cancer cell line was obtained from the State Key 
Laboratory of Molecular Oncology, Chinese Academy of Medical Sciences (Beijing, China). Human umbilical vein endothelial cells (HUVECs) were prepared and identified as previously described (11). Briefly, undamaged sections of fresh umbilical cords were cannulated, washed in PBS, perfused with trypsin and the detached cells were collected and resuspended in RMPI-1640 medium. A549 cells and HUVECs were cultured in RMPI-1640 medium supplemented with $10 \%(\mathrm{v} / \mathrm{v})$ foetal calf serum (FCS) at $37^{\circ} \mathrm{C}$ in $5 \% \mathrm{CO}_{2}$. A549 cell culture supernatant was used to stimulate HUVEC growth to obtain LCc-ECs as previously described (12). Cell culture reagents were purchased from Gibco (Carlsbad, CA, USA) and collagen type I was purchased from Sigma (St. Louis, MO, USA).

Cell viability assay. Briefly, LCc-ECs were plated in 96-well plates, allowed to attach, and made quiescent. Cells were treated with fasudil (Sigma) for $1 \mathrm{~h}$, and the number of viable cells was determined by addition of MTT solution for $2 \mathrm{~h}$ and measuring the absorbance at $630 \mathrm{~nm}$ as previously described (13). The experiments were performed in triplicate.

Cell migration assay. LCc-EC migration was analysed using the Boyden chamber assay. Briefly, LCc-ECs were resuspended at $1 \times 10^{4}$ cells $/ \mathrm{ml}$ in RMPI-1640 media without or with $0,20,40$ or $70 \mu \mathrm{mol}$ fasudil and $300 \mu \mathrm{l}$ aliquots of cell suspension were seeded into the matrigel-coated upper chamber of $8.0 \mu \mathrm{m}$ pore size Boyden chamber inserts (Becton-Dickinson, Franklin Lakes, NJ, USA). The chambers were inserted into the wells of 24-well plates coated with fibronectin and containing $700 \mu \mathrm{l}$ RMPI-1640 and $1 \mathrm{ml} \mathrm{NIH/3T3} \mathrm{fibroblast-conditioned} \mathrm{medium}$ as a chemoattractant. After $24 \mathrm{~h}$ incubation, the cells in the upper layer were swabbed with cotton, and cells which had migrated to the lower layer of the chamber were fixed in $95 \%$ ethanol and stained with hematoxylin. The number of cells which migrated through each membrane were counted using light microscopy (14).

Immunocytochemistry. The actin filaments in LCc-ECs were visualized by immunocytochemistry using a rhodamine-labelled anti-phalloidin antibody (Sigma). Briefly, fasudil-treated or untreated LCc-ECs were seeded on coverslips, fixed in $3.7 \%$ formaldehyde for $10 \mathrm{~min}$ at room temperature and incubated in $0.1 \%$ Triton X-100 in PBS (pH 7.4) for $5 \mathrm{~min}$ (13). To reduce non-specific background staining, the cells were incubated for $30 \mathrm{~min}$ with PBS containing $1 \%$ bovine serum albumin (BSA) prior to addition of antibody $(15,16)$. The images were recorded and analyzed using a Zeiss confocal photomicroscope LSM510 (Zeiss).

Reverse transcription PCR. Total RNA was isolated from LCc-ECs using TRIzol reagent (Invitrogen) according to the manufacturer's instructions and used for reverse transcription PCR following standard techniques with specific primers for TEM8 (forward: 5'-CGGATTGCGGACAGTAAGG-3'; reverse: 5'-GCCAGAACCACCGAGGAG-3'), TEM1 (forward: 5'-TCGAGTTATTGTAGCGAGGGACATG-3'; reverse: 5'-AGGTGGGCACCGGGTAGGGTAT-3'), RhoA (forward: 5'-GTGATTGTTGGTGATGGAGC-3'; reverse: 5'-CTCGTG GCCATCTCAAAAAC-3') and GAPDH as a control (forward:
5'-ACCACAGTCCATGCCATCAC-3'; reverse: 5'-TCCACCA CCCTGTTGCTGTA-3'). The PCR products were separated by electrophoresis on $1.5 \%$ agarose gels, visualized by ethidium bromide staining.

Western blot analysis. Untreated or fasudil treated LCc-ECs were lysed and centrifuged at $10,000 \mathrm{rpm}$ for $5 \mathrm{~min}$ at $4^{\circ} \mathrm{C}$ $(17,18)$. Protein concentrations were determined using the Bio-Rad protein assay system (Applied Biosystems, Foster City, USA) with BSA as a standard (19). The lysates were resolved by $12 \%$ SDS-PAGE and transferred to $0.2 \mu \mathrm{m}$ nitrocellulose membranes (20). Western blot analysis was performed using a monoclonal anti-human p-MLC antibody. Bands were visualized using autoradiography, the band intensities were quantified using BioMax Image Analysis software (Applied Biosystems) and protein expression levels were normalized to GAPDH.

Statistical analysis. Differences between groups were analyzed by one-way ANOVA using SPSS 13.0 (SPSS, Chicago, IL, USA); $\mathrm{P} \leq 0.05$ were considered statistically significant.

\section{Results}

Characteristics of LCc-ECs. Immunofluorescence staining confirmed that factor VIII related antigen (VIIIRAg) is expressed in LCc-ECs (Fig. 1A) showing that $\mathrm{LCc}$-ECs possess endothelial cell features. Using RT-PCR we detected that TEM1 (287 bp) and TEM 8 mRNA (462 bp), were expressed at high levels in LCc-ECs (Fig. 1B and C) compared to control HUVECs, indicating that LCc-ECs possess tumour-associated endothelial cell characteristics.

Fasudil reduces $L C c$-EC viability. Fasudil has been used to treat vascular spasms in the brain in Japan since $1995(9,10)$; however, the mechanism by which fasudil affects tumour angiogenesis has not been delineated. Therefore, we investigated the effect of fasudil on LCc-ECs in vitro. The MTT assay indicated that fasudil treatment significantly inhibited the viability of LCc-ECs in a dose-dependent manner after $96 \mathrm{~h}$ (Fig. 2). The $\mathrm{IC}_{50}$ for fasudil at $24 \mathrm{~h}$ in LCc-ECs was $84 \pm 4.6 \mu \mathrm{mol}$.

Fasudil inhibits LCc-EC migration. Cell migration is a critical step in the process of angiogenesis (21). Therefore, we evaluated the effects of fasudil treatment on the migration of LCc-ECs using in vitro migration assays. Treatment with fasudil significantly suppressed LCc-EC migration in a dosedependent manner (Fig. 3).

Fasudil treatment inhibits stress fibre formation in LCc-ECS. Cell migration begins with an initial protrusion or extension of the plasma membrane at the leading edge of the cell. The protrusion is driven by the polymerization of a network of cytoskeletal actin filaments and is stabilized through the formation of adhesive complexes (22). To investigate whether fasudil can prevent LCc-EC migration by affecting actin cytoskeletal organization, actin stress fibres were visualized by immunocytochemistry. Compared to control cells, treatment with fasudil led to stress fibre breakage and fracture in 
A

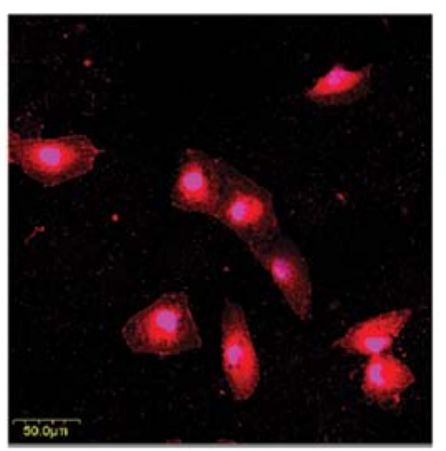

B

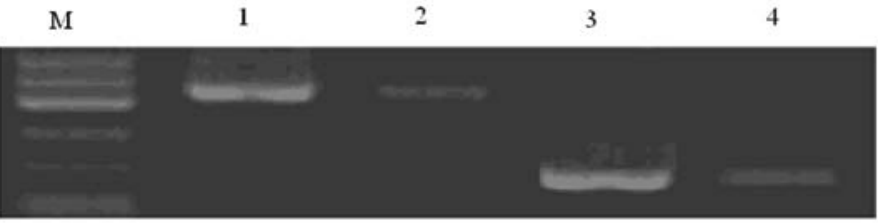

C

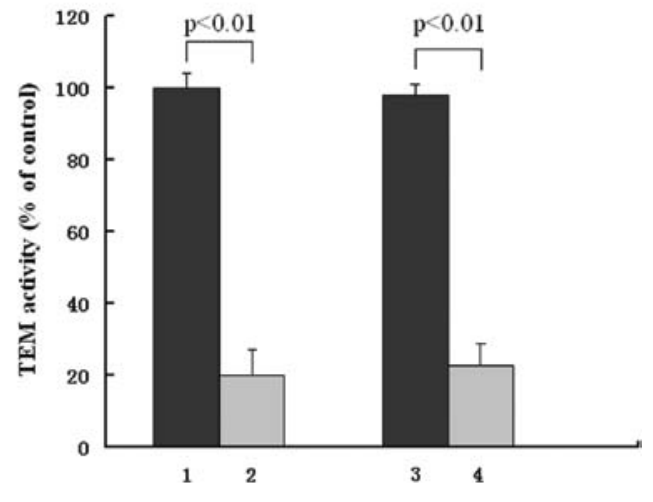

Figure 1. (A) Immunofluorescence staining of VIIIRAg in LCc-ECs using a primary anti-VIIIRAg antibody and a secondary fluorescein isothiocyanate (FITC)-conjugated antibody (red). VIIIRAg is visible as particles in the cytoplasm. (B and C) RT-PCR of TEM8 (460 bp) and TEM1 (290 bp) in control HUVECs and LCc-ECs. TEM 8 and TEM1 mRNA are both upregulated in LCc-ECs compared to HUVECs. M, DNA marker; lane 1, TEM8 in LCc-ECs; lane 2, TEM8 in HUVECs; lane 3, TEM1 in LCc-ECs; and lane 4, TEM1 in HUVECs.

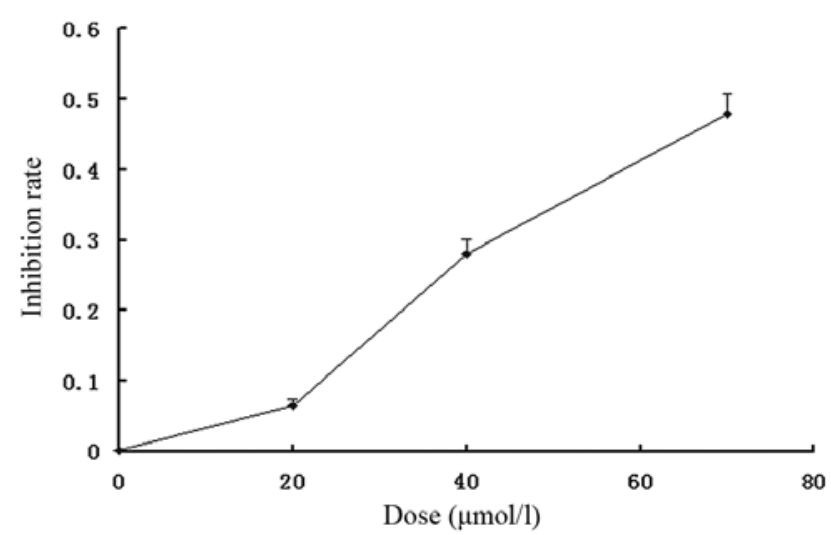

Figure 2. Fasudil treatment inhibits LCc-EC viability. The MTT assay was performed on LCc-ECs after treatment with the indicated concentrations of fasudil for $96 \mathrm{~h}$. The inhibition in cell viability is expressed as the ratio of the absorbance in cells treated with fasudil to control cells. Data are presented as the mean \pm standard error.

LCc-ECs (Fig. 4), indicating that fasudil impacts polymerisation of the cytoskeletal actin filament network.

Fasudil reduces expression of RhoA mRNA in LCc-ECS. We examined the expression of RhoA mRNA in LCc-ECs treated with $0,20,40$ and $70 \mu \mathrm{mol}$ fasudil using RT-PCR. Fig. 5 shows that the expression of $R h o A$ was significantly inhibited by fasudil in a dose-dependent manner.

Fasudil suppresses ROCK functionality in LCc-ECS. LCc-ECs were untreated or treated with fasudil for $1 \mathrm{~h}$ and western blot analysis was performed to determine the expression of the ROCK substrate p-MLC. Fasudil treatment significantly reduced the protein expression levels of p-MLC in a dose-dependent manner (Fig. 6).
A

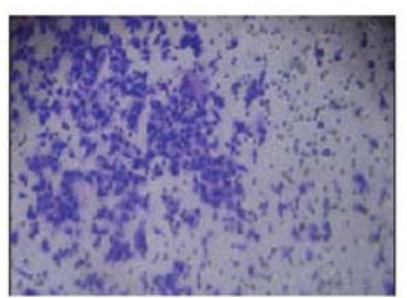

B

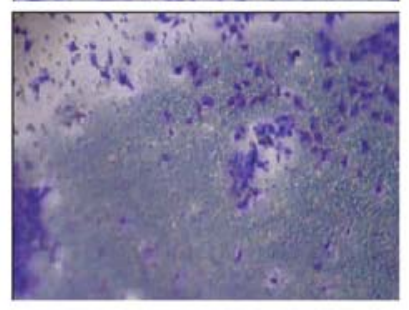

C

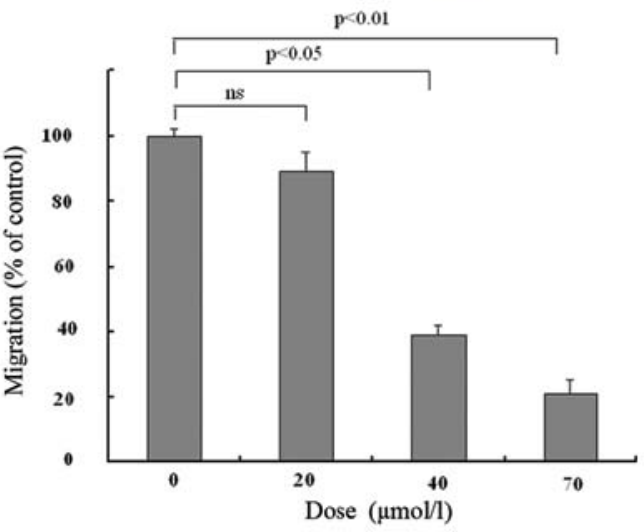

Figure 3. Fasudil treatment inhibits LCc-EC migration. LCc-EC migration was analyzed using the Boyden chamber assay using $8.0 \mu \mathrm{m}$ pore size inserts. Serum-starved untreated (A) or fasudil-pretreated (B) LCc-ECs were seeded into the upper chamber. After $24 \mathrm{~h}$, cells which had migrated to the lower layer of the membrane were fixed with $95 \%$ ethanol and stained with hematoxylin. The number of cells which migrated were counted under a light microscope. (C) Treatment with fasudil significantly suppressed LCc-EC migration in a dose-dependent manner. Results are representative of five independent experiments. 


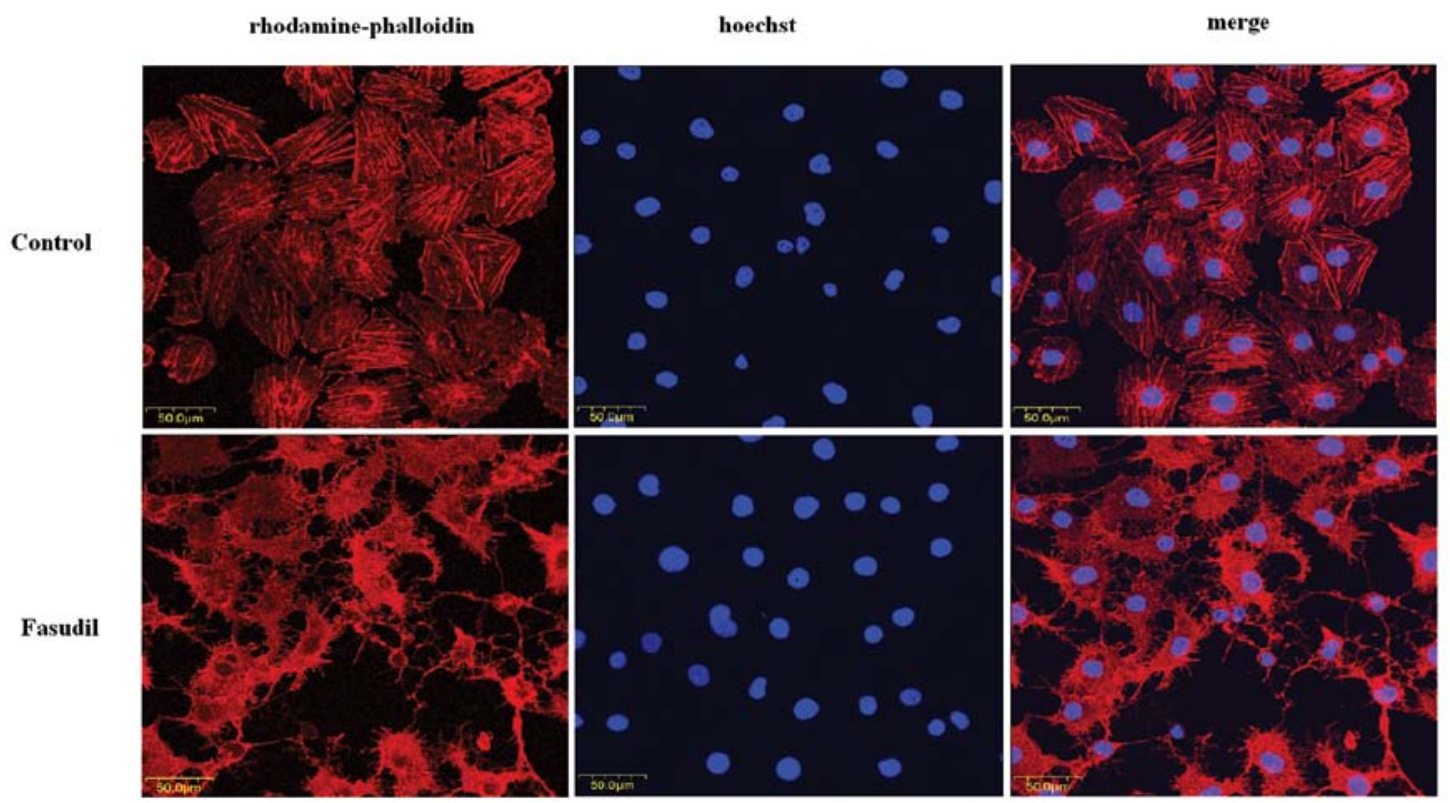

Figure 4. Fasudil treatment disrupts cytoskeletal actin filament integrity in LCc-ECs. Visualisation of the cytoskeletal actin filament in LCc-ECs using rhodamine-phalloidin staining (red); Hoechst was used as a nuclear counterstain (blue). The control group displayed cytoskeletal actin filament integrity, whereas treatment with $40 \mu \mathrm{mol}$ fasudil for $30 \mathrm{~min}$ lead to partial stress fibre breakage and fracture.

A

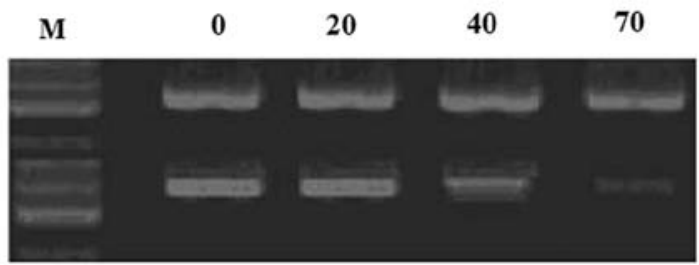

B

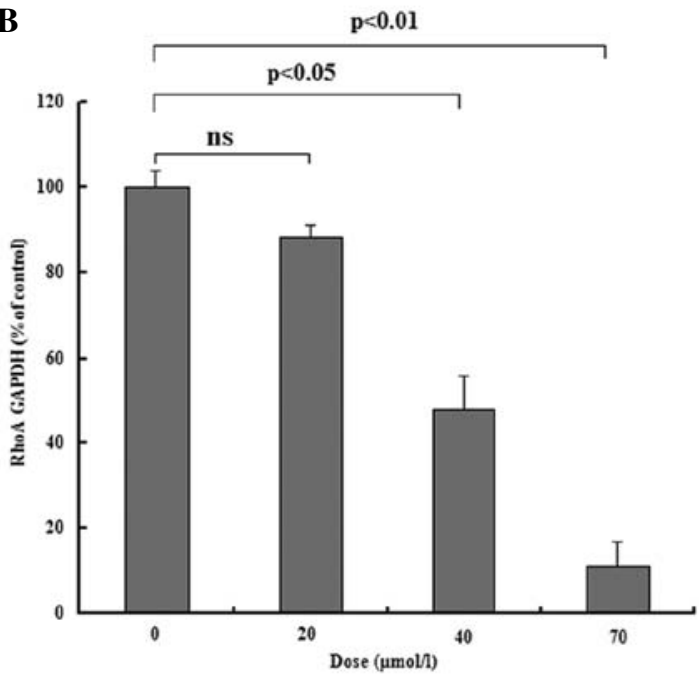

Figure 5. Fasudil treatment inhibits RhoA mRNA expression in LCc-ECs (A) RT-PCR of RhoA mRNA in LCc-ECs treated with 0, 20, 40 and $70 \mu \mathrm{mol}$ fasudil. (B) The expression of RhoA mRNA was significantly inhibited by fasudil in a dose-dependent manner in LCc-ECs.

\section{Discussion}

To date, there have been no studies on the effects and mechanism of action of fasudil in lung carcinoma-conditioned
A

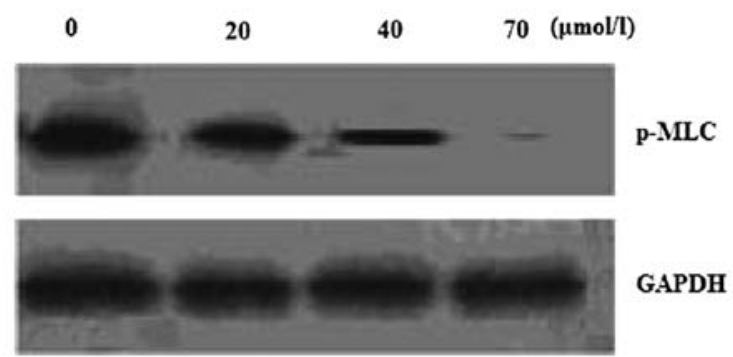

B

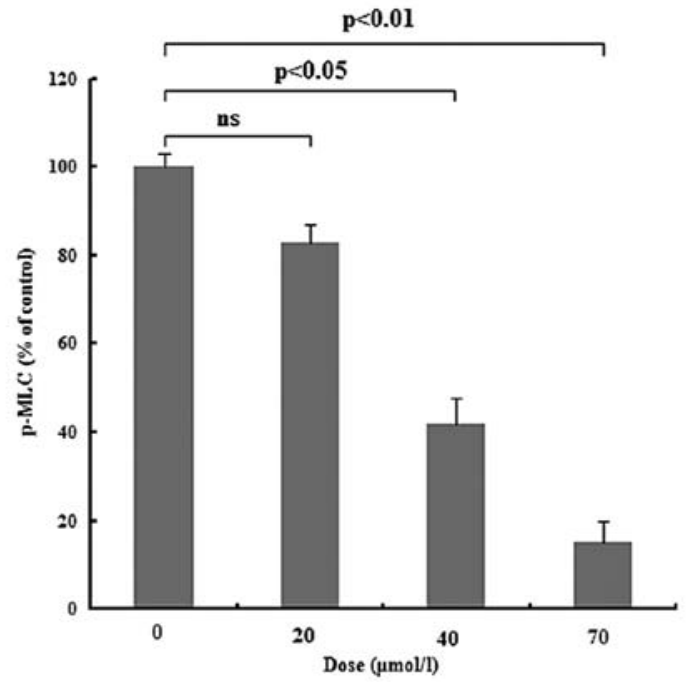

Figure 6. Fasudil treatment inhibits p-MLC expression in LCc-ECs. (A) Western blot analysis of p-MLC and GAPDH in LCc-ECs treated with 0 , 20,40 and $70 \mu$ mol fasudil. (B) p-MLC protein expression was significantly inhibited by fasudil in a dose-dependent manner in LCc-ECs.

endothelial cells (LCc-ECs). In this study we derived LCc-ECs from HUVECs using A549 lung cell carcinoma-conditioned 
media as a model of lung tumour endothelial cells. VIIIRAg staining of LCc-ECs confirmed that the cells were endothelial cells.

Tumour and normal vascular endothelial cells are different at the molecular level. TEM1 and TEM 8 mRNA expression characteristically increase in tumour-associated endothelial cells (4). We observed that TEMI and TEM 8 mRNA were significantly upregulated in LCc-ECs, compared to control HUVECs, indicating that LCc-ECs had acquired tumour endothelium characteristics.

In this study, fasudil treatment significantly inhibited the viability of LCc-ECs in a dose-dependent manner. It is well known that cancer progression and metastasis require cell motility (23), and cell migration is a key step in angiogenesis. Fasudil also markedly attenuated LCc-EC cell migration and lead to partial stress fibre breakage and fracture, resulting in a reduced in vitro invasive ability of LCc-ECs.

The GTPase RhoA mediates cell contraction by organizing actin filaments into stress fibres, and active RhoA is required for a variety of complex morphogenetic processes (11). RhoA activates ROCK, which plays an important role in the regulation of actin stress fibre organization (24). Treatment of LCc-ECs with fasudil resulted in altered stress fibre formation; therefore, it is likely that fasudil may affect RhoA/ ROCK signalling. Fasudil significantly and dose-dependently reduced expression of both RhoA mRNA and of the ROCK substrate p-MLC. These results indicate that the ability of fasudil to affect LCc-EC viability, invasion and actin polymerisation are linked to attenuated RhoA/ROCK activation and signalling. Further research is required to determine the mechanism by which fasudil inhibits RhoA expression and ROCK activation.

Previous experiments have indicated that RhoA/ROCK critically regulates VEGF-induced angiogenesis. Constitutively active RhoA can synergize with VEGF to stimulate angiogenesis (23), whereas dominant negative RhoA markedly inhibits VEGF-driven neovascularization (25). Thus, the function of RhoA is consistent with a possible role in vascular morphogenesis. ROCK may be a key player in ECs during angiogenesis (26). ROCK mediated angiogenesis plays an important role in tumour progression and metastasis, and is an attractive target for anticancer strategies.

In conclusion, this study demonstrates that fasudil significantly inhibits the viability and invasive ability of LCc-ECs, through a mechanism dependent on disrupted actin cytoskeleton polymerisation which is linked to reduced activation of RhoA/ROCK signalling. This study indicates that the antiangiogenic agent fasudil has potential applications for the treatment of cancer, especially lung adenocarcinoma.

\section{Acknowledgements}

This study was supported by the National Natural Science Foundation of China (no. 30973473/H1611).

\section{References}

1. Bauer PO, Wong HK, Oyama F, Goswami A, Okuno M, Kino Y, Miyazaki $\mathrm{H}$ and Nukina N: Inhibition of Rho kinases enhances the degradation of mutant huntingtin. J Biol Chem 284: 13153-13164, 2009.
2. Chang YW, Bean RR and Jakobi R: Targeting RhoA/Rho kinase and p21-activated kinase signaling to prevent cancer development and progression. Recent Pat Anticancer Drug Discov 4: 110-124, 2009.

3. Croft DR, Sahai E, Mavria G, Li S, Tsai J, Lee WM, Marshall CJ and Olson MF: Conditional ROCK activation in vivo induces tumor cell dissemination and angiogenesis. Cancer Res 64: 8994-9001, 2004.

4. St. Croix B, Rago C, Velculescu V, Traverso G, Romans KE, Montgomery E, Lal A, Riggins GJ, Lengauer C, Vogelstein B and Kinzler KW: Genes expressed in human tumor endothelium. Science 289: 1197-1202, 2000.

5. Samuel MS, Lopez JI, McGhee EJ, Daniel R, Croft DR, Strachan D, Timpson P, Munro J, Schroder E, Zhou J, et al: Actomyosin-mediated cellular tension drives increased tissue stiffness and $\beta$-catenin activation to induce epidermal hyperplasia and tumor growth. Cancer Cell 19: 776-791, 2011.

6. Demidem A, Morvan D and Madelmont JC: Bystander effects are induced by CENU treatment and associated with altered protein secretory activity of treated tumor cells: a relay for chemotherapy? Int J Cancer 119: 992-1004, 2006.

7. Sanz-Moreno V, Gaggioli C, Yeo M, Albrengues J, Wallberg F, Viros A, Hooper S, Mitter R, Féral CC, Cook M, et al: ROCK and JAK1 signaling cooperate to control actomyosin contractility in tumor cells and stroma. Cancer Cell 20: 229-245, 2011.

8. Basile JR, Gavard J and Gutkind JS: Plexin-B1 utilizes RhoA and Rho kinase to promote the integrin-dependent activation of Akt and ERK and endothelial cell motility. J Biol Chem 282: 34888-34895, 2007.

9. Ehrenreiter K, Kern F, Velamoor V, Meissl K, GalabovaKovacs G, Sibilia M and Baccarini M: Raf-1 addiction in Ras-induced skin carcinogenesis. Cancer Cell 16: 149-160, 2009.

10. Fu M, Zhang J, Tseng YH, Cui T, Zhu X, Xiao Y, Mou Y, De Leon H, Chang MM, Hamamori Y, et al: Rad GTPase attenuates vascular lesion formation by inhibition of vascular smooth muscle cell migration. Circulation 111: 1071-1077, 2005.

11. Yin L, Morishige K, Takahashi T, Hashimoto K, Ogata S, Tsutsumi S, Takata K, Ohta T, Kawagoe J, Takahashi K and Kurachi H: Fasudil inhibits vascular endothelial growth factorinduced angiogenesis in vitro and in vivo. Mol Cancer Ther 6: 1517-1525, 2007.

12. Jiang L, He Y and Jiang YG: Culture and cell biological characteristics of tumor-derived endothelial cells. Qingqing Medicine 38: 3102-3104, 2009.

13. Hata Y, Miura M, Nakao S, Kawahara S, Kita T and Ishibashi T: Antiangiogenic properties of fasudil, a potent Rho-Kinase inhibitor. Jpn J Ophthalmol 52: 16-23, 2008.

14. Kidera Y, Tsubaki M, Yamazoe Y, Shoji K, Nakamura H, Ogaki M, Satou T, Itoh T, Isozaki M, Kaneko J, et al: Reduction of lung metastasis, cell invasion, and adhesion in mouse melanoma by statin-induced blockade of the Rho/Rho-associated coiledcoil-containing protein kinase pathway. J Exp Clin Cancer Res 29: $127,2010$.

15. Lu Q, Longo FM, Zhou H, Massa SM and Chen YH: Signaling through Rho GTPase pathway as viable drug target. Curr Med Chem 16: 1355-1365, 2009.

16. Man K, Ng KT, Lo CM, Ho JW, Sun BS, Sun CK, Lee TK, Poon RT and Fan ST: Ischemia-reperfusion of small liver remnant promotes liver tumor growth and metastases - activation of cell invasion and migration pathways. Liver Transpl 13: 1669-1677, 2007.

17. Masamune A and Shimosegawa T: Signal transduction in pancreatic stellate cells. J Gastroenterol 44: 249-260, 2009.

18. Mavria G, Vercoulen Y, Yeo M, Paterson H, Karasarides M, Marais R, Bird D and Marshall CJ: ERK-MAPK signaling opposes Rho-kinase to promote endothelial cell survival and sprouting during angiogenesis. Cancer Cell 9: 33-44, 2006.

19. Somlyo AV, Phelps C, Dipierro C, Eto M, Read P, Barrett M, Gibson JJ, Burnitz MC, Myers C and Somlyo AP: Rho kinase and matrix metalloproteinase inhibitors cooperate to inhibit angiogenesis and growth of human prostate cancer xenotransplants. FASEB J 17: 223-234, 2003.

20. Sawada K, Morishige K, Mabuchi S, Ogata S, Kawase C, Sakata $M$ and Kimura T: In vitro and in vivo assays to analyze the contribution of Rho kinase in angiogenesis. Methods Enzymol 439: 395-412, 2008.

21. Takata K, Morishige K, Takahashi T, Hashimoto K, Tsutsumi S, Yin L, Ohta T, Kawagoe J, Takahashi K and Kurachi H: Fasudilinduced hypoxia-inducible factor-1alpha degradation disrupts a hypoxia-driven vascular endothelial growth factor autocrine mechanism in endothelial cells. Mol Cancer Ther 7: 1551-1561, 2008. 
22. Tang F, Wang D, Duan C, Huang D, Wu Y, Chen Y, Wang W, Xie C, Meng J, Wang L, et al: Berberine inhibits metastasis of nasopharyngeal carcinoma $5-8 \mathrm{~F}$ cells by targeting Rho kinasemediated Ezrin phosphorylation at threonine 567. J Biol Chem 284: 27456-27466, 2009.

23. Wong CC, Wong CM, Tung EK, Man K and Ng IO: Rho-kinase 2 is frequently overexpressed in hepatocellular carcinoma and involved in tumor invasion. Hepatology 49: 1583-1594, 2009.

24. Thoenes L and Gunther M: Novel approaches in anti-angiogenic treatment targeting endothelial F-actin: a new anti-angiogenic strategy? Curr Opin Mol Ther 10: 579-590, 2008.
25. Yamaguchi H, Shiraishi M, Fukami K, Tanabe A, Ikeda-Matsuo Y, Naito Y and Sasaki Y: MARCKS regulates lamellipodia formation induced by IGF-I via association with PIP2 and $\beta$-actin at membrane microdomains. J Cell Physiol 220: 748-755, 2009.

26. Zhao L, Xu G, Zhou J, Xing H, Wang S, Wu M, Lu YP and Ma D: The effect of RhoA on human umbilical vein endothelial cell migration and angiogenesis in vitro. Oncol Rep 15: 1147-1152, 2006. 